\title{
A comparison of wave mode identification techniques
}

\author{
S. N. Walker ${ }^{1}$, F. Sahraoui ${ }^{2}$, M. A. Balikhin ${ }^{1}$, G. Belmont ${ }^{2}$, J. L. Pinçon ${ }^{3}$, L. Rezeau ${ }^{2,4}$, H. Alleyne ${ }^{1}$, \\ N. Cornilleau-Wehrlin ${ }^{2}$, and M. André ${ }^{5}$ \\ ${ }^{1}$ Automatic Control \& Systems Engineering, The University of Sheffield, Mappin Street, Sheffield, S1 3JD, UK \\ ${ }^{2}$ Centre d'étude des Environnements Terrestre et Planétaires, 10-12 avenue de l'Europe, 78140 Vélizy, France \\ ${ }^{3}$ Lab. de Physique et Chimie de l'Environment, 3A Avenue de la Recherche Scientifique, 45071 Orleans cedex 2, France \\ ${ }^{4}$ also at Université de Pierre et Marie Curie, Paris, France \\ ${ }^{5}$ Swedish Institute for Space Physics, Box 537, 75121 Uppsala, Sweden
}

Received: 20 January 2004 - Revised: 22 April 2004 - Accepted: 18 May 2004 - Published: 7 September 2004

\begin{abstract}
The four point measurements available from the Cluster mission enable spatiotemporal effects in data sets to be resolved. One application of these multipoint measurements is the determination of the wave vectors and hence the identification of wave modes that exist within the plasma. Prior to multi-satellite missions, wave identification techniques were based upon the interpretation of observational data using theoretically defined relations. However, such techniques are limited by the quality of the data and the type of plasma model employed. With multipoint measurements, wave modes can be identified and their wave directions determined purely from the available observations. This paper takes two such methods, a phase differencing technique and $\mathrm{k}$-filtering and compares their results. It is shown that both methods can resolve the $\boldsymbol{k}$ vector for the dominant mirror mode present in the data. The phase differencing method shows that the nature of the wave environment is constantly changing and as such both methods result in an average picture of the wave environment in the period analysed. The k-filtering method is able to identify other modes that are present.
\end{abstract}

Key words. Space plasma physics (waves and instabilities; instruments and techniques) - Magnetospheric physics (magnetosheath)

\section{Introduction}

The characterization of the waves observed in space plasmas provides insight into the processes that are occurring within the plasma. One key aspect of this characterization is the identification of the low frequency wave modes that are present. These waves can play a pivotal role in the transfer and redistribution of energy within the particle populations that make up the plasma. In this paper, we compare two multi-spacecraft methods developed for the determina-

Correspondence to: S. N. Walker

(simon.walker@shef.ac.uk) tion of wave propagation direction $(\boldsymbol{k})$ and the wave propagation mode. The use of these methods is illustrated using an example of wave mode identification of fluctuations observed in the magnetosheath.

The approach taken to identify wave modes depends strongly on the number of simultaneous observations that are available. Until the advent of multi-spacecraft missions, wave mode identification had to be based on the observations of a single satellite. These single spacecraft methods were based upon the analysis of the relationship between the physical parameters observed in both field and plasma data which were expressed as a set of dimensionless transport ratios. Such analytic expressions (see Schwartz et al., 1996, for examples) were based on fluid models that may be unrealistic given the nature of the plasma region being investigated (Krauss-Varban et al., 1994). These methods have been applied to the identification of wave modes in the magnetosheath by Denton et al. (1995) and Song et al. (1994). However, they are limited to the identification of either monochromatic waves or the wave that carries the dominant energy.

The ability to use simultaneous data from two closely separated satellites enables the determination of the wave propagation direction and mode. This has be performed using either spectral/coherency analysis of the magnetic field components (Gleaves and Southwood, 1991) or magnetic and thermal pressures and a minimum variance analysis (MVA) (Song et al., 1992), together with the time delay information, or by computing the phase difference of the waves measured at each satellite (Balikhin and Gedalin, 1993; Dudok de Wit et al., 1995). The latter method results in the determination of the projection of the wave vector along the satellite separation direction. It is possible to reconstruct the complete wave vector (both magnitude and direction) in certain circumstances by employing a MVA to determine the actual wave propagation direction (Means, 1972) with respect to the satellite separation vector. However, the use of MVA imposes limitations on the use of this method, namely that it can only be applied to magnetic field data and that the wave 
in question should be monochromatic and elliptically polarized.

The use of data from four spacecraft has, until now, relied on a chance conjunction between satellites. Events of this nature have been reported by Russell et al. (1983), who presented observations of interplanetary shocks using the ISEE1,2 , and 3 spacecraft, together with either IMP-8 or Prognoz7 as the fourth. By analysing the time differences in relation to the spatial separations, the authors were able to determine the normals to the shocks studied. With the availability of routine four point measurements from the Cluster mission such techniques are now in common usage. In addition, new analytic techniques have been developed to exploit the four point measurements, to shed light onto the plasma wave environment. The three-dimensional measurements provide the ability to remove any spatiotemporal ambiguity that dogged earlier analysis techniques and to also provide the opportunity to identify multiple waves that can occur at the same frequency.

In this paper we compare two methods used for wave identification, namely a phase based method and k-filtering. Section 2 reviews these two methods. Section 3 describes the data sets used. Section 4 outlines the application of the dispersion technique to data collected by the Cluster satellites in the inner magnetosheath. A comparison of the results of both techniques are then presented in Sect. 5 .

\section{Methods}

The aim of this paper is to identify the main wave mode present in the period of data under consideration using the phase differencing method and then to compare the resulting wave vectors with those resulting from a second method, namely k-filtering. The following sections outline the application of the two methods.

Both methods assume that the measured waveform is the superposition of a number of plane waves. The general expression for this is given by

$$
\mathbf{B}(\boldsymbol{r}, t)=\sum_{\omega} \sum_{k} \mathbf{B}_{\omega, k} \exp i(\boldsymbol{k} \cdot \boldsymbol{r}-\omega t)+c . c .,
$$

where the quantity $\mathbf{B}_{\omega, k}$ is the amplitude of the wave associated with frequency $\omega$ and wave vector $\boldsymbol{k}$, and c.c. denotes the complex conjugate. The phase differencing method additionally assumes a unique wave vector for a given frequency which corresponds to the removal of the wave vector summation in Eq. (1). It is also assumed that the fields are stationary in time and homogeneous in space. In reality, such conditions are never met. However, these strict assumptions may be relaxed if we assume limited homogeneity in which the field is translation invariant over distances larger than the wavelengths being studied and that the signal is stationary over a period longer than that being studied.

\subsection{Phase differencing method}

The phase differencing method (Balikhin and Gedalin, 1993) has been successfully applied on a number of previous occasions (Balikhin et al., 1997; Chisham et al., 1999; Balikhin et al., 2001, 2002). It is solely reliant on the use of data collected by 4 (or more) closely-spaced spacecraft, such as that currently available from the Cluster mission. Since no assumptions such as those implied by the use of MVA are used in the analysis, the method is applicable not only to magnetic field data but also to fluctuations observed in the electric field or even particle parameters.

Frequency decomposition of the signals $\mathbf{B}(\boldsymbol{r}, t)$ is performed by wavelet decomposition techniques using a Morlet wavelet. This ensures good frequency resolution at the low frequencies that are of interest and a large number of frequency spectra that are used to accumulate statistics regarding the phase differences at each frequency. Hence, this method can be used successfully over short periods of data. However, for best results the lowest frequency considered should ensure that there are at least 4-6 wave periods within the data period being analysed.

If the same quantity is measured by two closely spaced satellites, $\alpha$ and $\beta$, the phase shift at a particular frequency between the two data sets is given by

$$
\begin{aligned}
\Delta \psi(\omega) & =\psi_{\alpha}(\omega)-\psi_{\beta}(\omega) \\
& =\left(\boldsymbol{k} \boldsymbol{r}_{\alpha}-\omega t\right)-\left(\boldsymbol{k} \boldsymbol{r}_{\beta}-\omega t\right) \\
& =|\boldsymbol{k}|\left|\boldsymbol{r}_{\alpha \beta}\right| \cos \left(\theta_{k r}\right),
\end{aligned}
$$

where $\boldsymbol{r}_{\alpha \beta}$ is the separation vector between the two satellites in question. The distribution of wave energy as a function of phase difference and frequency (referred to as an $\omega-k$ spectrum) may be created to show the relationship between the phase difference and frequency. The phase difference is related to the projection of the wave vector $\boldsymbol{k}$ along the satellite separation vector so that $\boldsymbol{r}_{\alpha \beta}$ may then be determined.

If data from only two satellites are available, other techniques such as MVA are required to determine the propagation direction (Balikhin et al., 1997; Chisham et al., 1999). However, the use of such methods severely limits the applicability of this phase differencing method for the determination of the wave vector.

With the advent of the 4 satellite Cluster mission, data are now available that are measured at four closely separated points in space. This enables the projection of the wave vector to be determined along three independent baselines (Balikhin et al., 2000; Bates et al., 2001; Balikhin et al., 2003). It is then possible to reconstruct the original $\boldsymbol{k}$ using a matrix inversion technique similar to that used by Russell et al. (1983) by solving the equation

$\boldsymbol{k} \cdot \mathbf{A}=\boldsymbol{k}_{r}$,

where $\mathbf{A}$ is a matrix consisting of the normalised satellite separation directions and $\boldsymbol{k}_{r}$ is a vector of the wave numbers projected onto the satellite separation directions. 
Once the $\boldsymbol{k}$ vector of the wave is known, it is possible to determine the plasma frame frequency $\left(\omega_{\text {plasma }}\right)$ of the wave in question from the frequency of the wave observed in the satellite frame $\omega_{o b s}$ and the plasma bulk velocity $\mathbf{V}$ by using the Doppler shift equation

$\omega_{\text {plasma }}=\omega_{o b s}-\boldsymbol{k} \cdot \mathbf{V}$.

\subsection{The k-filtering technique}

The k-filtering technique (Pinçon and Motschmann, 1998; Glassmeier et al., 2001) enables the identification of threedimensional structures in the wave field. It assumes that the fields that exist may be described by Eq. (1), together with the assumptions listed at the beginning of this section. The assumptions of limited homogeneity and weak stationarity are further justified because the k-filtering method is based upon the use of space-time correlation matrices. A further assumption not specifically related to the method is that the wave field should not contain waves of a length less than the interspacecraft separation. If this does occur, aliasing of the signals occurs, generating spurious results. This can, however, be overcome with a careful treatment of the results.

Developed in the context of space plasma physics by Pinçon and Lefeuvre (1992), the k-filtering method was applied for the first time to Cluster STAFF data by Sahraoui et al. (2003). For a given measured wave field $\mathbf{B}\left(t, \boldsymbol{r}_{\alpha}\right)$ at the positions $\boldsymbol{r}_{\alpha}(\alpha=1,2, \ldots)$, it enables an estimation of the fully 4D-energy distribution function $P(\omega, \boldsymbol{k})$ related to the field $\mathbf{B}$. In the context of this study, $\mathbf{B}$ represents the magnetic field measured by the STAFF-SC experiment on the four Cluster satellites. By considering plane wave decomposition Eq. (1), and assuming time stationarity during the studied interval, it is possible to obtain the frequency representation of the correlation matrix

$\overline{\overline{\mathbf{M}}}\left(\omega, \boldsymbol{r}_{\alpha}, \boldsymbol{r}_{\beta}\right)=<\mathbf{B}\left(\omega, \boldsymbol{r}_{\alpha}\right) \mathbf{B}^{*}\left(\omega, \boldsymbol{r}_{\beta}\right)>$,

where the symbol $<>$ denotes the time (or ensemble) average. Assuming as well a space homogeneity of the signal, it is possible to link the correlation matrix $\overline{\overline{\mathbf{M}}}$ with the energy distribution matrix $\overline{\overline{\mathbf{P}}}(\omega, \boldsymbol{k})$ by

$$
\begin{aligned}
\overline{\overline{\mathbf{M}}}\left(\omega, \boldsymbol{r}_{\alpha}, \boldsymbol{r}_{\beta}\right) & =<\mathbf{B}\left(\omega, \boldsymbol{r}_{\alpha}\right) \mathbf{B}^{*}\left(\omega, \boldsymbol{r}_{\beta}\right)> \\
& =\int \overline{\overline{\mathbf{P}}}(\omega, \boldsymbol{k}) e^{i \boldsymbol{k} \cdot \boldsymbol{r}_{\alpha \beta}} d \boldsymbol{k}
\end{aligned}
$$

where $\boldsymbol{r}_{\alpha \beta}=\boldsymbol{r}_{\alpha}-\boldsymbol{r}_{\beta}$. Inverting Eq. (6), i.e. estimating $\overline{\overline{\mathbf{P}}}(\omega, \boldsymbol{k})$ from $\overline{\overline{\mathbf{M}}}$, which is fully determined from the measurements, is a difficult task since the data are spatially undersampled. Overcoming this crucial problem is the fundamental aim of the k-filtering technique, which is grounded in the construction of a series of nonlinear filters. Each filter is related to a different $(\omega, \boldsymbol{k})$ pair and is built in such a way that only the energy associated with the angular frequency $\omega$ and the wave vector $\boldsymbol{k}$ is extracted from the data. While constructing these filters, any other information known on the data can be included. In our case, we introduce explicitly the constraint $\nabla . \mathbf{B} \equiv \boldsymbol{k} . \mathbf{B}=0$. The problem of the filter determination can be solved using the Lagrange multiplier method. For the details of the calculation, the reader is referred to Pinçon and Lefeuvre (1992) and Pinçon and Motschmann (1998). The final expression for the $P(\omega, \boldsymbol{k})$ estimation is obtained under the form

$$
P(\omega, \boldsymbol{k})=\operatorname{Tr}\{\overline{\overline{\mathbf{P}}}\}=\operatorname{Tr}\left\{\left[\overline{\overline{\mathbf{H}}}^{*}(\boldsymbol{k}) \cdot \overline{\overline{\mathbf{M}}}^{-1}(\omega) \cdot \overline{\overline{\mathbf{H}}}(\boldsymbol{k})\right]^{-1}\right\},
$$

where $\overline{\overline{\mathbf{H}}}$ is a geometrical matrix depending on the positions of the four satellites and taking into account the divergence free nature of the measured wave field. The quantity $P(\omega, \boldsymbol{k})$, which represents the magnetic energy distribution in Fourier space, is used to identify the propagating modes of this energy. We emphasize here that there is no limitation on the number of wave vectors $\boldsymbol{k}$ associated with a given frequency: a full $\boldsymbol{k}$-spectrum can be determined for each frequency $\omega$. This great advantage of the k-filtering method allows, as we shall see in Section 4.2, to identify several waves (i.e. several wave vectors) with different intensities for one given frequency.

\section{Observations}

The data were collected by the Cluster WEC STAFF search coil magnetometer (Cornilleau-Wehrlin et al., 1997) and the EFW double probe electric field instrument (Gustafsson et al., 1997). Both instruments were operating in a normal science mode, that generates a waveform of the electric/magnetic field in the frequency range up to $10 \mathrm{~Hz}$ and sampled at $25 \mathrm{~Hz}$. The magnetic field measurements from the STAFF search coil have been high-pass filtered using a cutoff frequency of $0.35 \mathrm{~Hz}$. This will ensure that there are no effects due to the spin of the spacecraft $(0.25 \mathrm{~Hz})$ in the data set. The EFW instrument measures the two components of the electric field in the spin plane of the satellite. The data are despun to remove the majority of the artifacts due to the spin of the satellites. Since the latitude of the spin axis of all four satellites (in the GSE frame) is $\approx-84^{\circ}$, this reference frame is very similar to the GSE frame rotated around the $\mathrm{X}$-axis by $180^{\circ}$. Due to the geometry of the spin axis of the satellites, the electric field booms may pass through the wake of the satellite, which leads to interference with a frequency of either twice or four times the spin frequency in the $E_{x}$ component of the electric field. Unfortunately, due to operational reasons, it was not possible to measure the electric field vector on satellite 1 . This implies that the method outlined above cannot be used to determine the wave vector of oscillations observed in the electric field. However, the remaining satellites can be used to check the projection of the wave vector on the separation vectors of satellites 2,3 , and 4.

This study uses data collected on 18 February 2002 between 05:34:00 and 05:36:44 UT, in order to determine the propagation direction $(\boldsymbol{k})$ of oscillations observed in the 


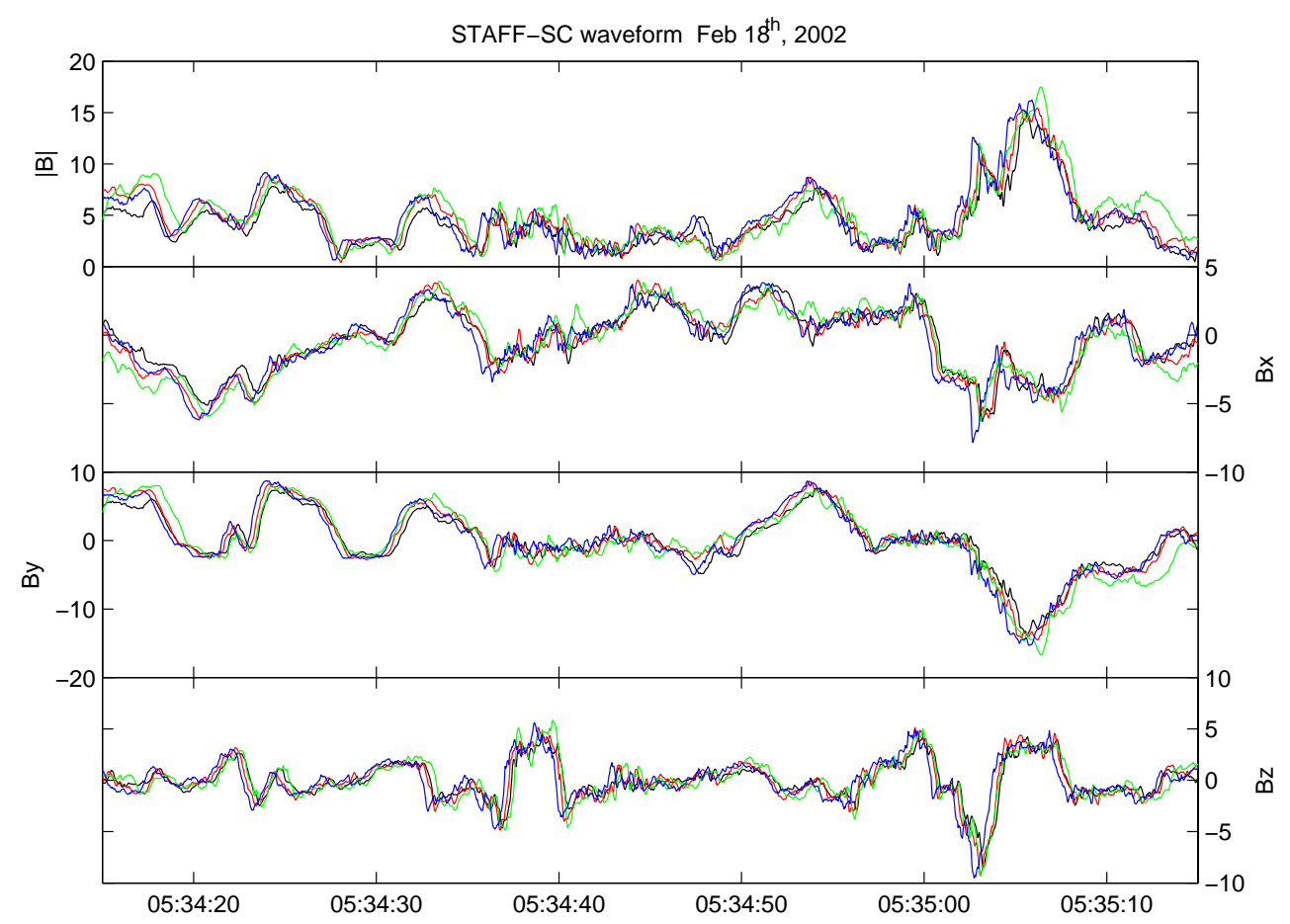

Fig. 1. The magnetic field measured by the STAFF search coil. From top to bottom, the panels show the field magnitude, and the X, Y, and $\mathrm{Z}$ components in the GSE frame. The colored lines represent data from Cluster 1 (black), 2 (red), 3 (green), and 4 (blue).

electric and magnetic fields. During this period, the satellites were on an outbound trajectory, crossing the magnetopause and entering the magnetosheath $\approx 04: 59$ UT. Their position was approximately $(5.6,4.6,8.4) R_{E}$ GSE, approximately $0.8 R_{E}$ outside the model magnetopause of Roelof and Sibeck (1993), and the interspacecraft separation was between 94 and $104 \mathrm{~km}$. The configuration of the four spacecraft is virtually identical to that of a regular tetrahedron.

Two commonly used geometrical parameters that are used to describe the 3-D shape of the spacecraft configuration are the elongation E and planarity P (Robert et al., 1998). For the ideal 3-D configuration (a regular tetrahedron) the values of $\mathrm{E}$ and $\mathrm{P}$ are close to zero. In our case, we have $\mathrm{P}=0.04$ and $E=0.08$. Thus, the satellites are in a position that is well suited to determine the propagation direction of the observed waves. During this period, the plasma bulk velocity was $(-181,25,129) \mathrm{kms}^{-1}$.

Figure 1 shows the waveform of the magnetic field measured by the STAFF search coil magnetometer during the period 05:34:15 and 05:35:15 UT on 18 February 2002. The four panels show (from top to bottom) the magnitude of the magnetic field, together with the GSE X, Y, and Z components for the four Cluster satellites, using the colour scheme black, red, green, and blue for the satellites Cluster 1, 2, 3, and 4 , respectively. This corresponds to approximately the first minute of the total period and will be the portion of data used in the wave vector determination process using the phase differencing method as opposed to the whole period which was used in the k-filtering analysis. This period was chosen because of the relatively stable nature of the observed waves and the consistency of the $\omega-k$ spectra. During the rest of the period the $\omega-k$ spectra are not as clear, as further branches in the dispersion tend to appear and then disappear. This shows the changeability of the nature of the waves observed in this short period. This will be addressed further in Section 5. During this first minute, the FFT spectra show evidence for small amplitude fluctuations occurring in the frequency range $0.35-1.1 \mathrm{~Hz}$. The objective of this paper is to determine the wave vector of the waves observed in this frequency range and to identify their mode of propagation.

The corresponding data measured by the EFW instruments on satellites 2, 3, and 4 are shown in Fig. 2. The waveforms show that the $\mathrm{X}$ component of the signal measured on satellites 3 and 4 is dominated by an oscillation whose period corresponds to half of the spin period of the satellites. The $\mathrm{Y}$ components show a much smaller effect due to the spin rotation and thus will be used as a comparison for the results obtained from the analysis of the STAFF search coil data. These spin effects also make it impossible to calculate the time lag of the waveform as it propagates from one satellite to the next.

\section{Results}

\subsection{Phase differencing}

The $\omega-k$ dispersions calculated using data from STAFF SC for the time period 05:34:15-05:35:15 UT is shown in 


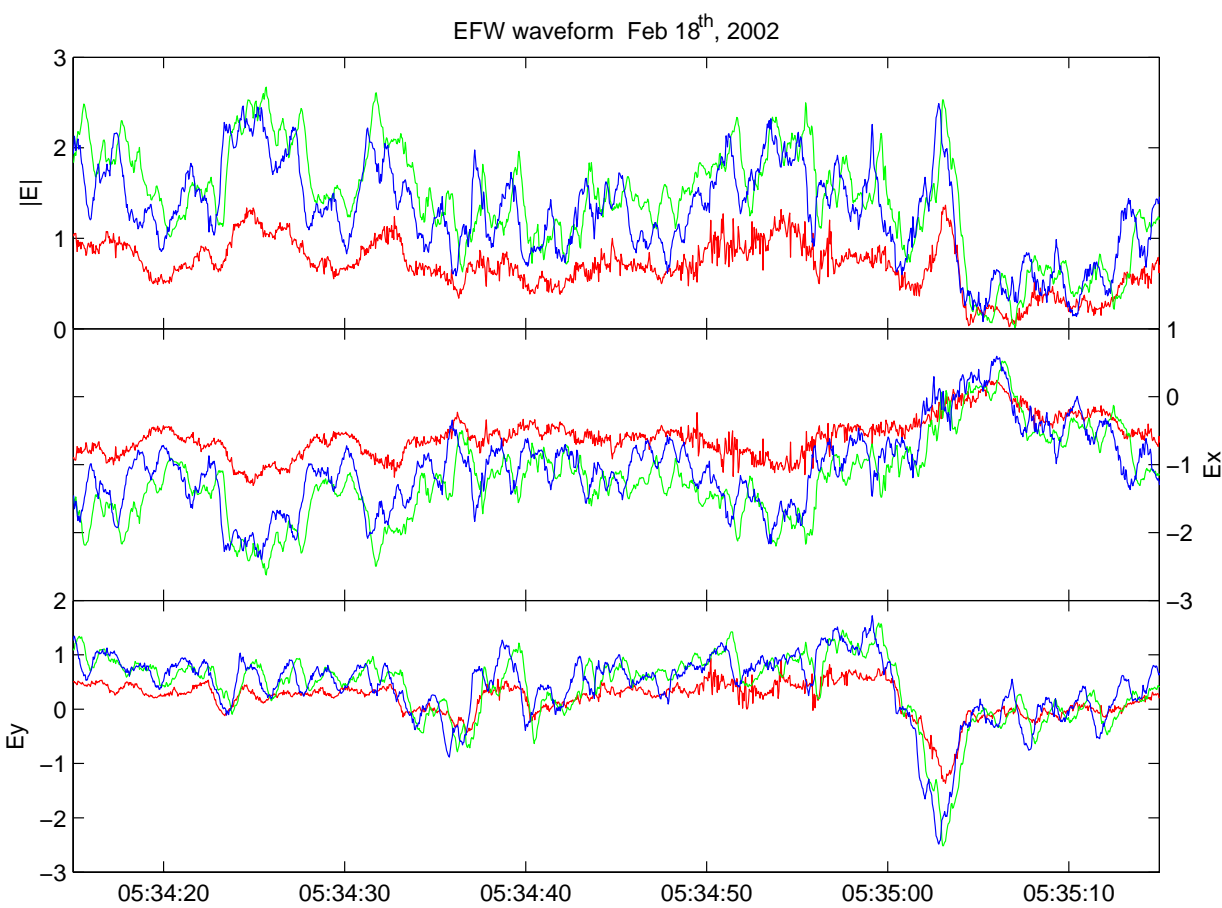

Fig. 2. The electric field measured by the EFW instruments. From top to bottom, the panels show the spin plane field magnitude, and the $X$ and Y components. The coloured lines represent data from Cluster 2 (red), 3 (green), and 4 (blue). The X component seen on satellites 3 and 4 show strong evidence of fluctuations occurring at twice the spin frequency which is probably the result of one of the probe pairs passing through the wake of the satellites.

Fig. 3. All three dispersions (using satellite combinations 14, 24, and 34) show a distinctive linear feature that exhibits an increase in the phase difference of the fluctuations with increasing wave frequency. For each of these plots the positive slope of the branch indicates that the waves are propagating from the second satellite towards the first. Thus, the results shown in Fig. 3 imply that the waves are observed by satellite 4 first, followed by whichever of the other satellites was used to generate the $\omega-k$ dispersion plots. A comparison of the dispersions calculated for all satellite combinations shows that during the period studied, the waves cross the satellites in the order 4,2 , followed by 3 and 1 , with no obvious preference. This result generally agrees with the crossing order observed in the waveform data shown in Fig. 1, in which satellite 4 (indicated by the blue line) is always the first to observe any fluctuation in the field.

Due to the ambiguity in the determination of the phase, the spectra are periodic with a period $2 \pi$. The determination of the correct branch of the dispersion may be made by examination of the waveforms. During this period the similarity of the waveforms implies that all satellites see the same wave with time differences less than the inverse frequency. Hence, the correct branch to examine in the dispersion plot is the central one, i.e. the branch that converges to the point $f=0 \mathrm{~Hz}, \Delta \psi / \boldsymbol{r}=0$. Thus, the $2 n \pi$ ambiguity in the dispersion has been removed. This also implies that the wavelength of the waves being studied is much greater than the interspacecraft separation distance.
The white crosses mark the peaks in the dispersion curve at frequencies of $0.37,0.44,0.62$, and $1.1 \mathrm{~Hz}$. The horizontal lines through the crosses represent the width of the peak in the dispersion at the frequencies studied. The $\boldsymbol{k}$ vector of the waves at each of these frequencies was determined and the results shown in Table 1. The typical error in the estimation of the projection of the wave vector along the satellite separation distance from the plots shown in Fig. 3 is of the order of $\pm 1.7 \times 10^{-3} \mathrm{~km}^{-1}$. This translates to an accuracy of around $10^{\circ}$ in the determination of the direction of $\boldsymbol{k}$. The direction of the $\boldsymbol{k}$-vectors differs considerably from the minimum variance direction computed using the same data period, with the difference being $\approx 40^{\circ}$. This is understandable since mirror modes have a linear polarisation and so the similarity between the intermediate and minimum eigenvalues implies that the minimum variance direction is not well defined.

Now that the wave vector direction has been determined it is possible to identify the propagation mode of the waves. Using the Doppler Eq. (4) the plasma frame frequency $\omega_{\text {plasma }}$ may be determined. The results are shown in Table 1 . The phase velocity of the waves $V_{\text {phase }}$ was determined to be of the order of $200 \mathrm{kms}^{-1}$. The angle between the wave vector and the plasma bulk velocity is $\approx 25^{\circ}$, which implies that the phase velocity of the wave is comparable with the projection of the plasma bulk velocity in the direction of the wave vector. Finally, the angle of propagation with respect to the magnetic field is of the order of $85^{\circ}$. This evidence points towards the waves propagating in the mirror mode. 

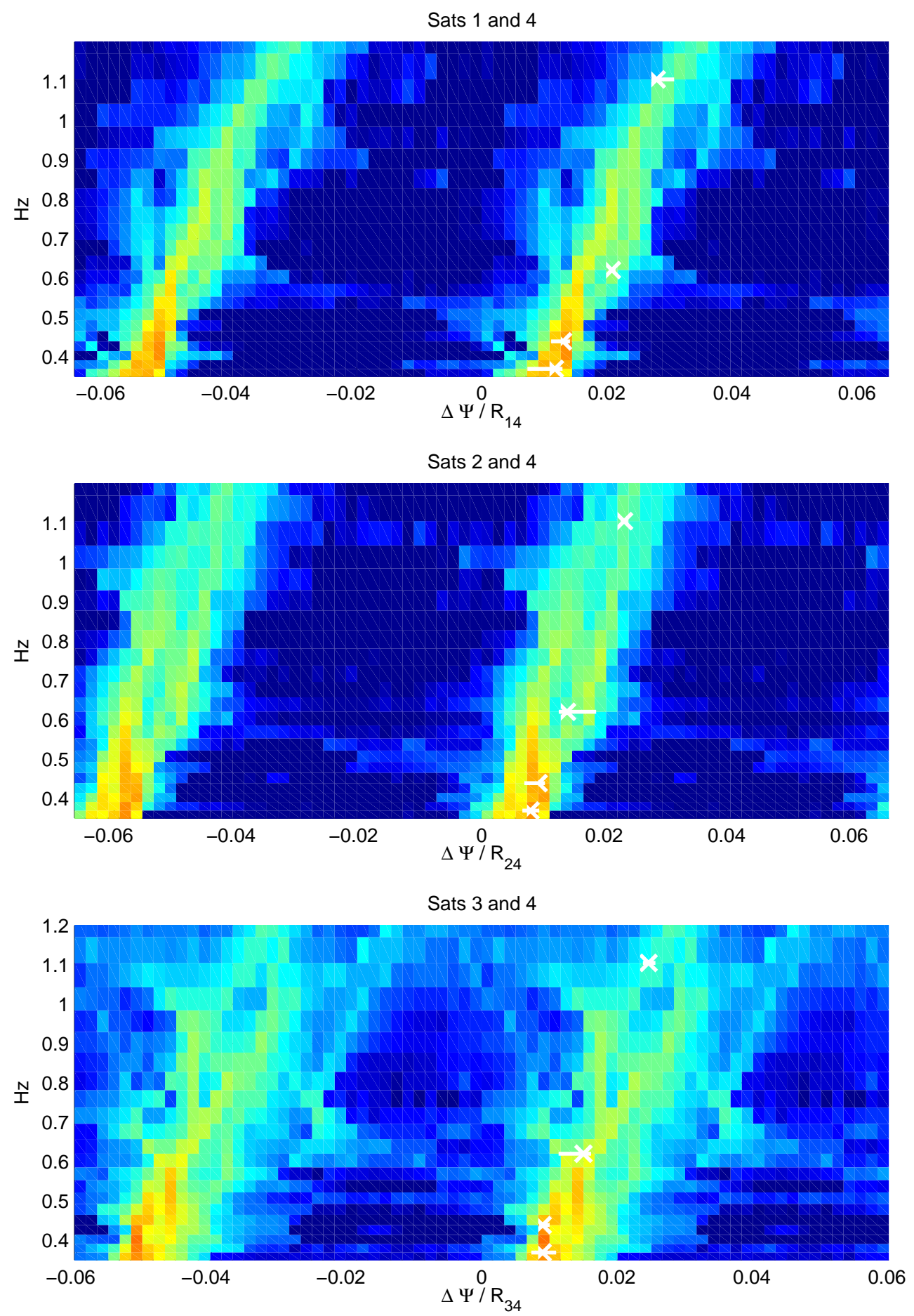

Fig. 3. The $\omega-k$ joint spectrogram computed using STAFF SC data for satellite pairs 14,24 , and 34 .

As a way of validating these $\boldsymbol{k}$ vectors, electric field data from EFW was also used to determine the wave vector. However, as has already been pointed out, since the electric field is not available from satellite 1 , it is impossible to calculate the full wave vector $\boldsymbol{k}$ using the method described above. It is, however, possible to calculate the projection of the wave vector along the satellite separation directions. Fig- ure 2 shows the electric field waveform measured by the EFW instruments on satellites 2, 3, and 4 (coloured red, green and blue, respectively). The bottom panel shows the magnitude of the electric field measured in the spin plane of each satellite. The middle and top panels show the $E_{x}$ and $E_{y}$ spin plane components. The waveforms from satellites 3 and 4 are almost identical in nature. This is clearly evident 
Table 1. Wave vectors $(\boldsymbol{k})$ computed for the four frequencies being investigated using the phase differencing method.

\begin{tabular}{llllll}
\hline $\begin{array}{l}f_{\text {sat }} \\
(\mathrm{Hz})\end{array}$ & $\begin{array}{l}f_{\text {plas }} \\
(\mathrm{Hz})\end{array}$ & $\begin{array}{l}k_{x} \\
(\mathrm{rd} / \mathrm{km})\end{array}$ & $\begin{array}{l}k_{y} \\
(\mathrm{rd} / \mathrm{km})\end{array}$ & $\begin{array}{l}k_{z} \\
(\mathrm{rd} / \mathrm{km})\end{array}$ & $\begin{array}{l}\|\boldsymbol{k}\| \\
(\mathrm{rd} / \mathrm{km})\end{array}$ \\
\hline 0.37 & 0.03 & -0.009959 & -0.003494 & 0.006239 & 0.0122 \\
0.44 & 0.01 & -0.009969 & -0.00462 & 0.007797 & 0.01347 \\
0.62 & 0.13 & -0.016833 & -0.006143 & 0.0119 & 0.02151 \\
1.10 & 0.2 & -0.0253 & -0.01283 & 0.01264 & 0.03106 \\
\hline
\end{tabular}

Table 2. Wave vectors $(\boldsymbol{k})$ projected along the satellite separation vectors. The frequency used was $0.37 \mathrm{~Hz}$. The values were determined using a phase-based method with both STAFF-SC $\left(\boldsymbol{k}_{S T ~ A F F}\right)$ and EFW electric field $\left(\boldsymbol{k}_{E F W}\right)$ and by applying the k-filtering method to the STAFF-SC data set.

\begin{tabular}{lccl}
\hline & \multicolumn{3}{c}{ Satellite pair } \\
& 14 & 24 & 34 \\
\hline $\boldsymbol{k}_{S T A F F}$ & -0.01087 & -0.00739 & 0.00837 \\
$\boldsymbol{k}_{E F W}$ & - & -0.0074 & 0.0084 \\
$\boldsymbol{k}_{k-f i l t}$ & -0.00943 & -0.00556 & 0.01055 \\
\hline
\end{tabular}

in the components of the electric field, in which fluctuations are observed first by Cluster 4 (blue) followed after a short time delay of $0.4 \mathrm{~s}$ by Cluster 3 . Some of these oscillations have a period of around $2 \mathrm{~s}$, which relates to twice the spacecraft spin frequency. In our analysis, we will only consider frequencies that are not multiples of the spacecraft spin frequency and so should not be influenced by spin effects. The waveform from Cluster 2 is similar to that of satellites 3 and 4 , however, it does not show the fluctuations repeated to the spin period as strongly.

The $\omega-k$ dispersion of the electric field Y component for satellite pairs 2 and 4 (top panel) and 3 and 4 (bottom panel) is shown in Fig. 4. The horizontal band in the frequency range $0.4-0.6 \mathrm{~Hz}$ is the result of the spin effects due to the individual probes passing through the shadow of the satellite. From Fig. 4 it is possible to determine the value of the $\boldsymbol{k}$ vector projected onto the satellite separation directions 24 , and 34. Table 2 lists the projections of the wave vector along the satellite separation directions used in the analysis above. The $\boldsymbol{k}$ directions determined using the phase difference method with STAFF-SC and EFW data give identical results. These results are very similar to those determined using the k-filtering method.
Table 3. The computed characteristics of the most intense identified wave for the four studied frequencies using the k-filtering technique. The wave vectors are in the quasi-perpendicular direction, $\theta_{\left(\boldsymbol{k}, \boldsymbol{B}_{\mathbf{0}}\right)} \sim-87^{\circ}$.

\begin{tabular}{ccccc}
\hline $\begin{array}{c}f_{\text {sat }} \\
\mathrm{Hz}\end{array}$ & $\begin{array}{c}k_{x} \\
\mathrm{rd} / \mathrm{km}\end{array}$ & $\begin{array}{c}k_{y} \\
\mathrm{rd} / \mathrm{km}\end{array}$ & $\begin{array}{c}k_{z} \\
\mathrm{rd} / \mathrm{km}\end{array}$ & $\begin{array}{c}\|k\| \\
\mathrm{rd} / \mathrm{km}\end{array}$ \\
\hline 0.37 & -0.01097 & -0.00236 & 0.00528 & 0.01241 \\
0.44 & -0.01241 & -0.00279 & 0.00529 & 0.01378 \\
0.61 & -0.01671 & -0.00404 & 0.00682 & 0.01849 \\
1.12 & -0.03065 & -0.00941 & 0.01438 & 0.03514 \\
\hline
\end{tabular}

\section{2 k-filtering}

Most of the results that will be presented in this section are those obtained by Sahraoui et al. (2003) using the k-filtering technique. Instead of providing the details of the identified waves, as was done in the original paper, we will give a general overview of the physics embedded in frequency range $[0.35,1.2] \mathrm{Hz}$. Let us first recall the principle of the study: for each frequency $\omega_{0}$ in the studied spectrum we calculate the related spatial spectrum $P\left(\omega_{0}, \boldsymbol{k}\right)$. We are particularly interested in the localization of the maxima of the energy in the $\boldsymbol{k}$ domain. The experimentally determined dispersion relations $\left(\omega_{0}, \boldsymbol{k}\right)$ are then compared to those derived from linear, low-frequency plasma wave theory and calculated using the measured plasma parameters (ions temperature, density, plasma beta, ...). The details of this comparison can be consulted in Sahraoui et al. (2003).

Since the results of the original analysis presented in Sahraoui et al. (2003) contain a small error related to the transformation of the STAFF-SC data from the GSE frame to one that is aligned with the local magnetic field (MFA), we take the opportunity, in the context of the present comparative study, of giving the corrected characteristics of the identified waves in the GSE frame. In Table 3, we summarize the results obtained concerning the most intense wave identified for four frequencies ranging from 0.35 to $1.2 \mathrm{~Hz}$. All these maxima can be interpreted as belonging to a unique spread wave packet, which has wave vectors in the quasiperpendicular direction with respect to the ambient magnetic field. Moreover, the corresponding frequencies in the plasma frame are almost zero. These two arguments make most probable the attribution of this wave to a mirror mode.

In addition to the mirror mode, the k-filtering technique enables us also to identify other weaker waves for each of the previous studied frequencies, corresponding to slow, Alfvén and cyclotron modes. An example of such a coexistence between several waves for one given frequency is shown in Fig. 5, which represents the isocontours of the function $P(\omega, \boldsymbol{k})$ in the $\left(k_{x}, k_{y}\right)$ plane for the frequency $f=0.61 \mathrm{~Hz}$. A cyclotron mode close to the second gyroharmonic $f_{\text {plasma }}=0.71 \mathrm{~Hz} \sim 2 f_{c i}$ (bottom panel) is identified 

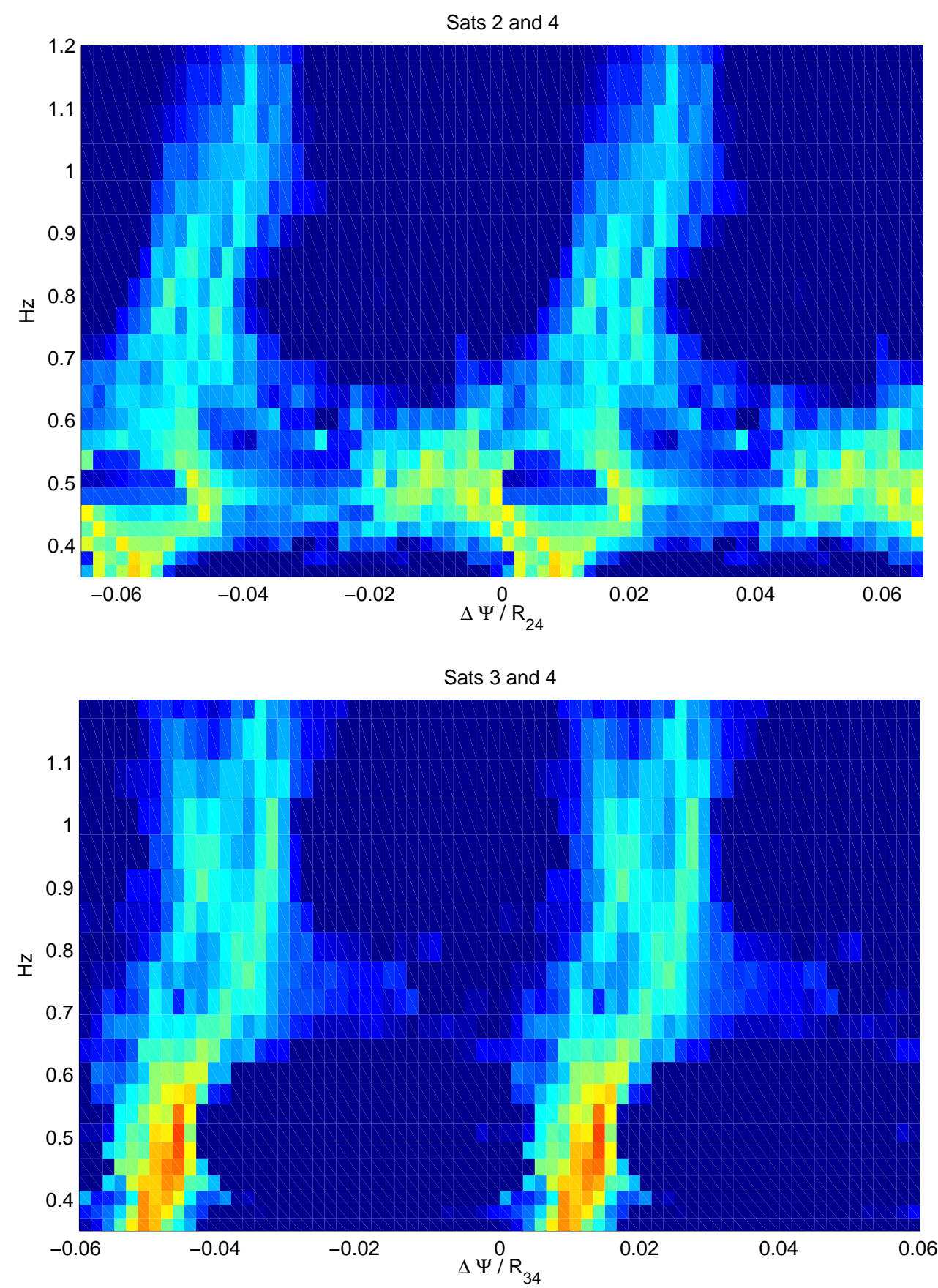

Fig. 4. The $\omega-k$ joint spectrogram computed using EFW electric field data.

simultaneously with a mirror mode (top panel). For a given frequency and $k_{z}$ value, a mode is clearly identified when its theoretical curve in the $\left(k_{x}, k_{y}\right)$ plane lies in close proximity to the observed magnetic energy peak. We also notice that the superposition of the slow, Alfvén, and mirror dispersion curves in Fig. 5 (top panel) reflects the degeneracy of these modes at low frequencies in the plasma frame $(\approx 0 \mathrm{~Hz})$ and particularly for this quasi-perpendicular direction of propagation $\left(\approx 87^{\circ}\right)$. The characteristics of the first secondary maximum for each of the studied frequencies are summarized in Table 4. The main result that one may retain from the present analysis is that up to $0.7 \mathrm{~Hz}$ in the satellite frame, slow and Alfvén modes coexist with the dominant mirror mode (see Fig. 5). For higher frequencies, proton gyroharmonics are also identified which seem to have energies comparable to that of the mirror mode. This tendency of the mirror mode to lose its dominant character is illustrated in Table 4 by comparing in column "I" its energy to that of the first secondary maximum. For the frequency $f=1.12 \mathrm{~Hz}$ the "rest" of the mirror mode has the same energy than that of the sixth gyroharmonic. 
Table 4. Using the k-filtering technique other waves with lower intensities are identified for the four studied frequencies. Only the first secondary maxima are given here. In Column "I" are given their intensities compared to that of the dominant wave for the corresponding frequency. $f_{\text {plasma }}$ is the relative frequency in the plasma frame, $f_{c i}=0.33 \mathrm{~Hz}$ is the proton gyrofrequency.

\begin{tabular}{llccll}
\hline $\begin{array}{l}f_{\text {sat }} \\
\mathrm{Hz}\end{array}$ & $\begin{array}{l}\|k\| \\
\mathrm{rd} / \mathrm{km}\end{array}$ & $\theta_{\left(\boldsymbol{k}, \boldsymbol{B}_{\mathbf{0}}\right)}$ & $\begin{array}{c}f_{\text {plasma }} \\
\mathrm{Hz}\end{array}$ & Mode & $\mathrm{I}$ \\
\hline 0.37 & 0.01914 & $-30^{\circ}$ & $-0.14 \sim 0.4 f_{c i}$ & Slow & $12 \%$ \\
0.44 & 0.02326 & $-45^{\circ}$ & -0.29 & Alfvén $\sim f_{c i}$ & $21 \%$ \\
0.61 & 0.04246 & $44^{\circ}$ & 0.71 & Cycl. $\sim 2 f_{c i}$ & $33 \%$ \\
1.12 & 0.04553 & $-57^{\circ}$ & 1.99 & Cycl. $\sim 6 f_{c i}$ & $98 \%$ \\
\hline
\end{tabular}

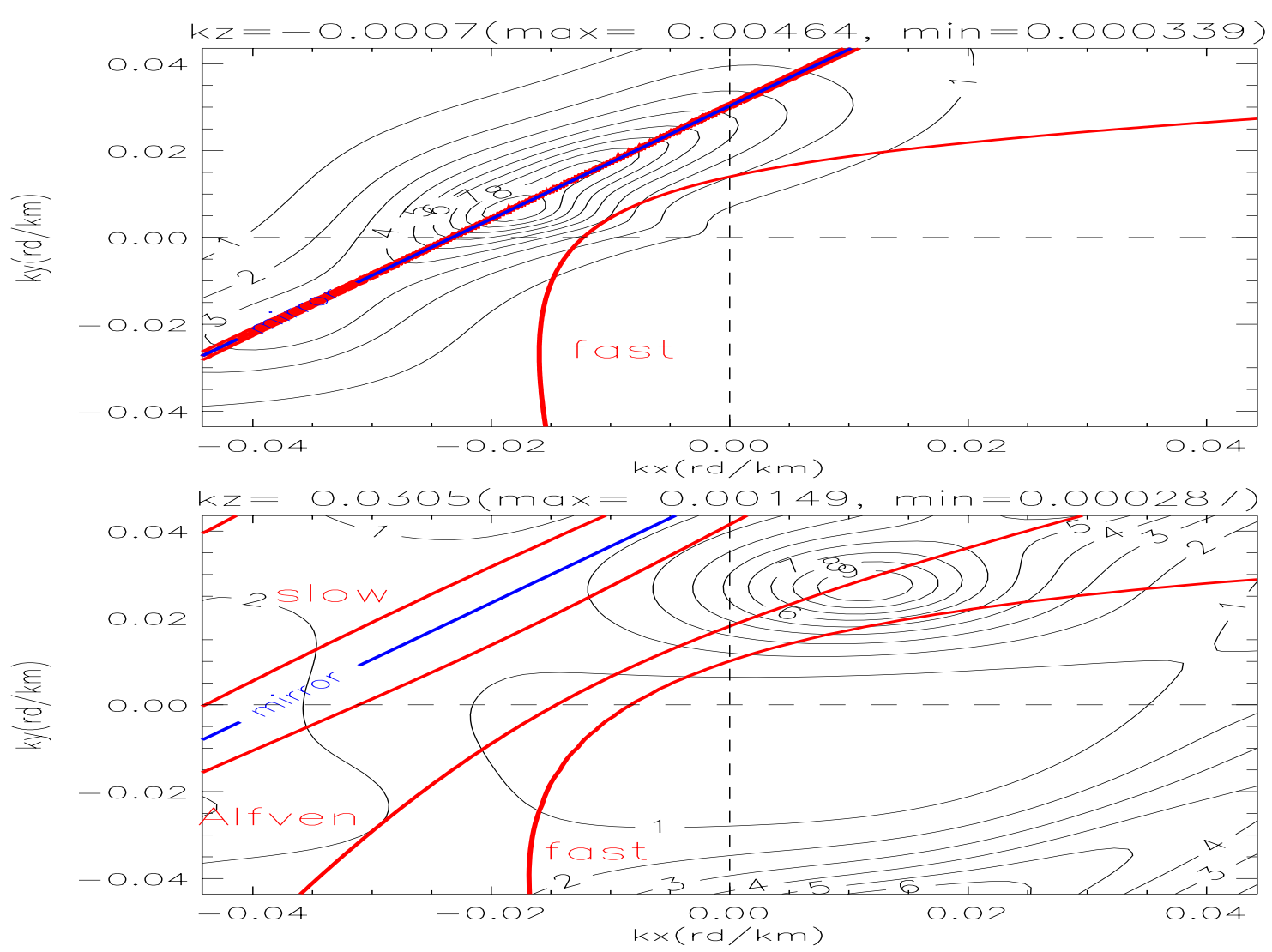

Fig. 5. Superposition of the experimental magnetic energy (thin black lines) with the theoretical dispersion relations of the LF modes (colored thick lines) in the satellite frame for the frequency $0.61 \mathrm{~Hz}$. The blue line is the Doppler shift $\omega=\boldsymbol{k}$.v. Two main peaks are identified: a mirror mode (top panel) and a cyclotron harmonic mode $\left(f_{\text {plasma }}=0.71 \mathrm{~Hz} \sim 2 f_{c i}\right)$, which seems to have a dispersion close to the classical MHD Alfvén mode (bottom panel).

\section{Discussion}

The analysis of the strongest signal seen in the $\omega-k$ dispersion plots obtained using the phase differencing method shows evidence for these waves propagating in the mirror mode. The results of analysing both the magnetic field data from the STAFF SC instrument and electric field data from EFW produce very similar values for the projection of the wave vector along the satellite separation directions. Thus, we can be fairly confident with the value of $\boldsymbol{k}$.
The nature of the waves in this period has also been studied by Sahraoui et al. (2003) using the k-filtering technique. Their results show that there are a number of different wave modes operating, the most dominant of which is the mirror mode. The projections of the wave vector along the satellite separation directions, as determined by the k-filtering method for the frequency $0.37 \mathrm{~Hz}$ are also shown in Table 2 as a comparison for those determined using the dispersion technique. 
(a) 053415-053630

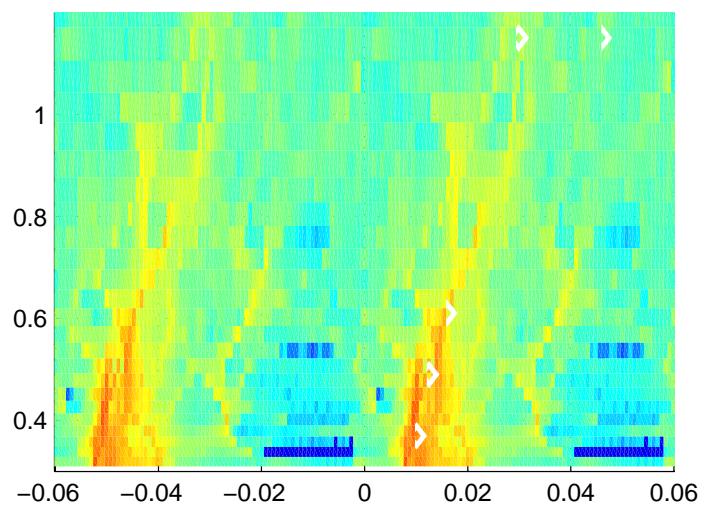

(c) 053440-053455

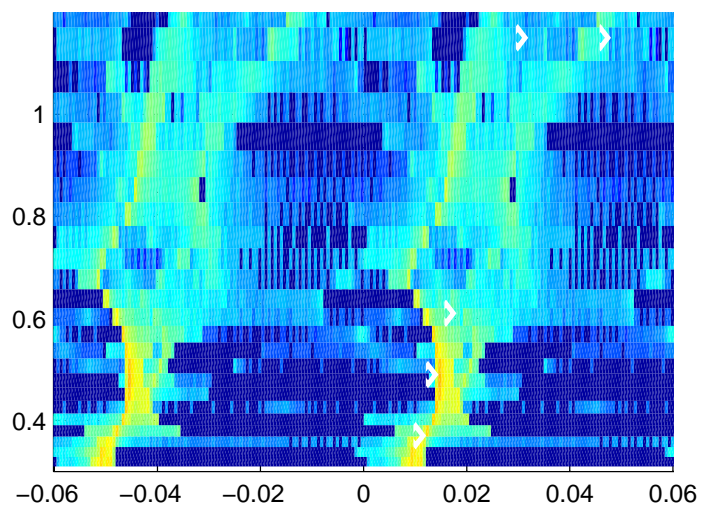

(b) 053415-053430

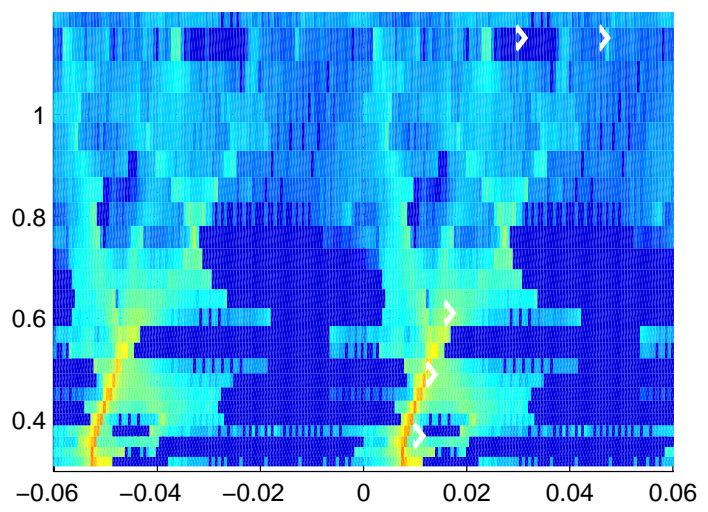

(d) 053555-053610

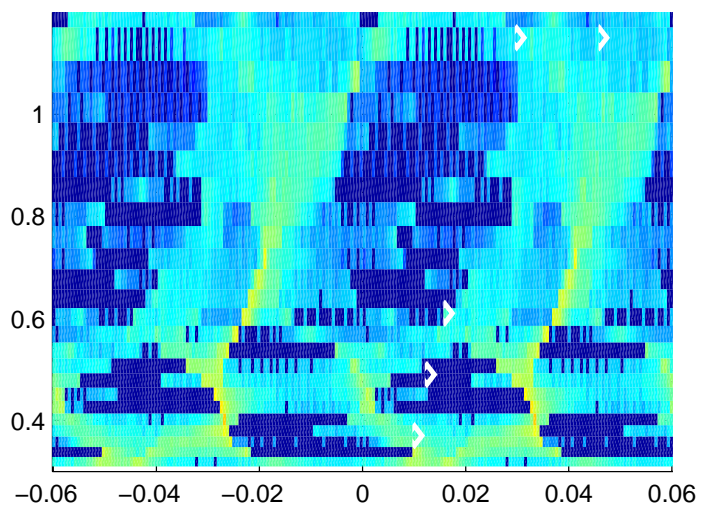

Fig. 6. A set of snapshots of the $\omega-k$ dispersion. Panel (a) is calculated using data covering the period 05:34:15-05:36:30 UT. The other snapshots are computed using 15-s worth of data, beginning at different times within the period under study. In all cases data from satellites 3 and 4 were used. Superimposed onto each plot are the projections of the wave vectors determined using the k-filtering method along the satellite separation vectors. The white diamonds superimposed onto each plot are the projections of the wave vectors associated with mirror modes determined using the $\mathrm{k}$-filtering method.

For these mirror mode waves, the values of $|\boldsymbol{k}|$ are very similar. From the $\omega-k$ dispersion $|\boldsymbol{k}|=0.0122 \mathrm{~km}^{-1}$ whilst the k-filtering method results in $|\boldsymbol{k}|=0.0124 \mathrm{~km}^{-1}$. Moreover, the actual directions of the $\boldsymbol{k}$ vectors differ only by $\approx 8^{\circ}$, which is less than the error that was estimated from the dispersion method mentioned above. However, this error could be larger for higher frequencies because of the presence of other modes, with comparable energies. In fact, in addition to identifying the mirror mode, the k-filtering technique also shows the existence of other modes such as an Alfvén, slow and cyclotron mode. The phase differencing technique cannot be used to resolve the presence of more than one wave mode unless (as in the above example) one mode dominates the dispersion.

It was mentioned in the previous section that during the period 05:34:00-05:36:44 UT, the nature of the waves is highly changeable. Figure 6 shows a set of snapshots of the $\omega-k$ dispersion determined for different time ranges within this period for the satellites 3 and 4 . It should be noted that these plots were computed using a much higher resolution in $\boldsymbol{k}$ space than those shown in Figs. 3 and 4. In each case the horizontal axis represents the phase difference projected along the separation vector between satellites 3 and 4 . The range of $\Delta \psi$ represented is $\pm 2 \pi$, corresponding to limits of $\pm 0.06 \mathrm{~km}^{-1}$. As a result, the multiple branches represent the $2 n \pi$ ambiguity in the determination of the phase difference. Panel (a) was calculated using STAFF SC data covering the period 05:34:15-05:36:30 UT. This plot represents an average of the dispersions for all waves measured during this period. Examining the structure of the dispersion branches it is observed that there are a number of different dispersion curves. The main dispersion branch corresponds to values of $\Delta \psi / \mathbf{r}_{34} \approx 0.01-0.015$ at a frequency of $0.35 \mathrm{~Hz}$. At frequencies between $0.4-0.5 \mathrm{~Hz}$ this section of the dispersion branch appears to be double peaked in $\boldsymbol{k}$ space and splits into two at frequencies above $\approx 0.8 \mathrm{~Hz}$. To the right of this main peak in the dispersion there is a second branch that appears in the frequency range $0.35-0.8 \mathrm{~Hz}$. This section of the dispersion curve has an intensity greater than an order of magnitude less than the main dispersion curve and will correspond to a different wave mode. The mirror mode wave vectors determined by Sahraoui et al. (2003) have been used to calculate 
the wave vector projections along the satellite separation directions. These results have then been superimposed on these dispersion curves and are represented by the white diamonds. This demonstrates the agreement presented in previous sections.

If instead of considering the whole period of data under study we concentrate rather on shorter sub-intervals, the pattern of dispersions varies, usually simplifying itself in that there are fewer dispersion curves featured within the branches of the plot. Panel (b) of Fig. 6 shows the $\omega-k$ spectrogram calculated for a $15-\mathrm{s}$ period, beginning at 05:34:15 UT. At frequencies below $0.7 \mathrm{~Hz}$ the dispersion curve is well defined and correlates extremely well with the mirror mode determined using k-filtering. At the highest frequency considered there seems to be little evidence for the continuation of the mirror modes. This may be because the waves have an extremely low amplitude and so are difficult to distinguish from the background. As was noted in Sect. 4 the features in the wave dispersions around this period were fairly consistent and so were used to compute the wave vector of the mirror modes. Panel (c) shows a 15-s snapshot of the dispersion, beginning at 05:34:40 UT. At low frequencies, the $n=0$ dispersion branch still closely resembles the mirror mode dispersion. At the uppermost frequency considered by Sahraoui et al. (2003) (1.15 Hz), the mirror mode waves correspond to a pair of branches seen in the $\omega-k$ dispersion plot. At the end of the period studied in Sahraoui et al. (2003), the dispersion, shown in panel (d), is completely different in nature. The mirror mode waves previously identified have become virtually non-existent whilst a new branch, identified as the lower amplitude curve to the right of the main dispersive branch in panel (a), has become the dominant wave mode over the majority of the frequency range being considered. The isolation and identification of these other dispersion branches will form the basis of a future investigation.

\section{Conclusions}

In this report we have used two different methods (k-filtering and phase differencing) to determine the wave modes present within the magnetosheath in the vicinity of the magnetopause. Both methods have evolved to make use of the multipoint measurements made available from the Cluster mission.

The results show that both analysis techniques identify the dominant wave mode present in the data as a mirror mode. The $\boldsymbol{k}$-vectors determined are also in reasonable agreement. Both methods also show the existence of other modes. The results presented indicate that the wave environment is constantly changing and hence the assumption that the signals are stationary during the whole period being analysed may be incorrect. However, it is possible to isolate shorter time periods when this assumption will hold true.

Regarding the k-filtering technique, it can be shown that the main consequence of this flickering is an underestimation of the energy value associated with the various modes. The determined wave vectors remain unaffected. This has been confirmed by performing the k-filtering analysis on shorter time intervals. The different methodologies used for conversion from the time to the frequency domain show both advantages and disadvantages in the techniques. Using a wavelet transform based on the Morlet wavelet, the dispersion based method can produce good clear results on a shorter period of data than the k-filtering technique, however, the Fourier transform used in k-filtering enables the energy density of the individual waves to be determined. This information is not available when using the Morlet wavelet transform.

It was shown that the phase differencing method works best when only one wave mode is present or when one wave mode dominates the wave environment. Multiple modes result in a number of dispersion curves and so the wave vector directions are currently unresolvable. Methods to resolve this problem are currently being pursued. In contrast, the k-filtering technique can resolve multiple waves within the plasma.

From the analysis presented above and the results of Sahraoui et al. (2003), it is clear that both wave identification methods find mirror mode waves present at low frequencies.

Acknowledgements. SNW is funded by PPARC through grant PPA/G/R/1999/00487. FS is funded by Centre National des Etudes Spatial (CNES).

Topical Editor T. Pulkkinen thanks S. P. Gary and another referee for their help in evaluating this paper.

\section{References}

Balikhin, M., Bates, I., and Dunlop, M.: Minimum Variance Free Identification of the Composition of Space Plasma Turbulence and Nonlinear Processes in it, in: Proceedings of the Cluster-II Workshop Multiscale/Multipoint Plasma Measurements, 22-24 Sept. 1999, Imperial College, London, UK, edited by Balough, A., Escoubet, C. P., and Harris, R. A., vol. ESA SP-449, 287, 2000 .

Balikhin, M. A. and Gedalin, M. E.: Comparative analysis of different methods for distinguishing temporal and spatial variations, in: Proc. of START Conf., Aussois, France, vol. ESA WPP 047, 183-187, 1993.

Balikhin, M. A., Dudok de Wit, T., Woolliscroft, L. J. C., Walker, S. N., Alleyne, H., Krasnosel'skikh, V., Mier-Jedrzejowicz, W. A. C., and Baumjohann, W.: Experimental determination of the dispersion of waves observed upstream of a quasiperpendicular shock, Geophys. Res. Lett., 24, 787-790, 1997.

Balikhin, M. A., Schwartz, S., Walker, S. N., Alleyne, H. S. K., Dunlop, M., and Lühr, H.: Dual spacecraft observations of standing waves in the magnetosheath, J. Geophys. Res., 106, 25395 25 408, 2001.

Balikhin, M. A., Walker, S. N., Alleyne, H. S., and Bates, I.: Minimum variance free wave identification: Application to Cluster electric field measurements in the magnetosheath, Tech. Rep. 852, ACSE, University of Sheffield, 2002.

Balikhin, M. A., Pokhotelov, O. A., Walker, S. N., Amata, E., Andre, M., Dunlop, M., and Alleyne, H. S. K.: Minimum variance free wave identification: Application to Cluster electric field data in the magnetosheath, Geophys. Res. Lett., 30, 15-1, 2003. 
Bates, I., Balikhin, M. A., Alleyne, H., and Andre, M.: Minimum variance free determination of magnetosheath wave propagation vectors, in: Les Woolliscroft Memorial Conference Sheffield Space Plasma Meeting: Multipoint measurements versus theory, vol. ESA SP-492, European Space Agency, Paris, 133, 2001.

Chisham, G., Schwartz, S. J., Balikhin, M., and Dunlop, M. W.: AMPTE observations of mirror mode waves in the Magnetosheath: Wavevector determination, J. Geophys. Res., 104, 437, 1999.

Cornilleau-Wehrlin, N., Chauveau, P., Louis, S., Meyer, A., Nappa, J. M., Perraut, S., Rezeau, L., Robert, P., Roux, A., De Villedary, C., de Conchy, Y., Friel, L., Harvey, C. C., Hubert, D., Lacombe, C., Manning, R., Wouters, F., Lefeuvre, F., Parrot, M., Pinçon, J. L., Poirier, B., Kofman, W., Louarn, P., and the STAFF Investigator Team: The Cluster Spatio-Temporal Analysis of Field Fluctuations (Staff) Experiment, Sp. Sci. Rev., 79, 107-136, 1997.

Denton, R. E., Gary, S. P., Li, X., Anderson, B. J., Labelle, J. W., and Lessard, M.: Low-frequency fluctuations in the magnetosheath near the magnetopause, J. Geophys. Res., 100, 56655679, 1995.

Dudok de Wit, T., Krasnosel'skikh, V. V., Bale, S. D., Dunlop, M. W., Lühr, H., Schwartz, S. J., and Woolliscroft, L. J. C.: Determination of dispersion relations in quasi-stationary plasma turbulence using dual satellite data, Geophys. Res. Lett., 22, 2653-2656, 1995.

Glassmeier, K.-H., Motschmann, U., Dunlop, M., Balogh, A., Acuña, M. H., Carr, C., Musmann, G., Fornaçon, K.-H., Schweda, K., Vogt, J., Georgescu, E., and Buchert, S.: Cluster as a wave telescope: first results from the fluxgate Magnetometer, Ann. Geophys., 19, 1439, 2001.

Gleaves, D. G. and Southwood, D. J.: Magnetohydrodynamic fluctuations in the earth's magnetosheath at 1500 LT - ISEE 1 and ISEE 2, J. Geophys. Res., 96, 129-142, 1991.

Gustafsson, G., Bostrøm, R., Holback, B., Holmgren, G., Lundgren, A., Stasiewicz, K., Åéhlen, L., Mozer, F. S., Pankow, D., Harvey, P., Berg, P., Ulrich, R., Pedersen, A., Schmidt, R., Butler, A., Fransen, A. W. C., Klinge, D., Thomsen, M., Falthammar, C.-G., Lindqvist, P.-A., Christenson, S., Holtet, J., Lybekk, B., Sten, T. A., Tanskanen, P., Lappalainen, K., and Wygant, J.: The electric field and wave experiment for the Cluster mission, Sp. Sci. Rev., 79, 137-156, 1997.
Krauss-Varban, D., Omidi, N., and Quest, K. B.: Mode properties of low frequency waves: Kinetic Theory versus Hall-MHD, J. Geophys. Res., 99, 5987-6009, 1994.

Means, J. D.: Use of the three-dimensional covariance matrix in analyzing the polarization properties of plane waves, J. Geophys. Res., 77, 5551-5559, 1972.

Pinçon, J. L. and Lefeuvre, F.: The application of the generalized Capon method to the analysis of a turbulent field in space plasma: Experimental constraints, J. Atmos. Terr. Phys, 54, 1237-1247, 1992.

Pinçon, J.-L. and Motschmann, U.: Multispacecraft filtering:general framework, in Analysis methods for multi-spacecraft data, Int. Space Sci. Inst., Bern, Switzerland, 65-78, 1998.

Robert, P., Roux, A., Harvey, C. C., Dunlop, M. W., Daly, P. W., and Glassmeier, K.-H.: Tetrahedron geometric factors, in: Analysis methods for multi-spacecraft data, Int. Space Sci. Inst., Bern, Switzerland, 323-348, 1998.

Roelof, E. C. and Sibeck, D. C.: Magnetopause shape as a bivariate function of interplanetary magnetic field $b_{z}$ and solar wind dynamic pressure, J. Geophys. Res., 98, 21 421-21 450, 1993.

Russell, C. T., Mellot, M. M., Smith, E. J., and King, J. H.: Multiple spacecraft observations on interplanetary shocks: Four spacecraft determination of shock normals, J. Geophys. Res., 88, 4739-4748, 1983.

Sahraoui, F., Pinçon, J. L., Belmont, G., Rezeau, L., CornilleauWehrlin, N., Robrt, P., Mellul, L., Bosqued, J. M., Balogh, A., Canu, P., and Chanteur, G.: ULF wave identification in the magnetosheath: The k-filtering technique applied to Cluster II data, J. Geophys. Res., 108(A9), 1335, 2003.

Schwartz, S. J., Burgess, D., and Moses, J. J.: Low frequency waves in the Earth's magnetosheath: Present status, Ann. Geophys., 14, 1134-1150, 1996.

Song, P., Russell, C. T., and Thomsen, M. F.: Waves in the Inner Magnetosheath: A Case Study, Geophys. Res. Lett., 19, 21912194, 1992.

Song, P., Russell, C. T., and Gary, S. P.: Identification of lowfrequency fluctuations in the terrestrial magnetosheath, J. Geophys. Res., 99, 6011-6025, 1994. 